\title{
Maxillofacial Reconstruction in a Pediatric Patient with Osteosarcoma
}

\author{
R Lopez Fernandez ${ }^{1}$, J Tellez Rodriguez ${ }^{1}$, J Shalkow Klincovstein ${ }^{2}$, R Bologna Molina ${ }^{3}$, R González Gonzá- \\ lez ${ }^{3}$ \\ ${ }^{1}$ Department of Maxillofacial Surgery. National Institute of Pediatrics, Mexico City. \\ ${ }^{2}$ Department of Surgical Oncology. National Institute of Pediatrics, Mexico City. \\ ${ }^{3}$ Research Department, School of Dentistry, Juarez University of the Durango State, Durango, Mexico.
}

Correspondence:

Predio Canoas $s / n$

Col. Los Angeles,

C.P 34070 ,

Duango, Durango, México.

email:bt_patologia@yahoo.com.mx

Lopez Fernandez R, Tellez Rodriguez J, Shalkow Klincovstein J, Bologna Molina R, González González R. Maxillofacial Reconstruction in a Pediatric Patient with Osteosarcoma. J Clin Exp Dent. 2011;3(Suppl1):e369-72. $\mathrm{http} / / /$ www.medicinaoral.com/odo/volumenes/v3iSuppl1/jcedv3iSuppl1p369.pdf

Article Number: $50502 \quad$ http://www medicinaoral.com/odo/indice.htm (C) Medicina Oral S. L. C.I.F. B 96689336 - eISSN: 1989-5488

eMail: jced@jced.es

\begin{abstract}
Osteosarcoma is a bone tumor that consists of malignant cells that produce immature bone. Is a bone tumor that develops during periods of rapid growth in adolescents and young adults. It is the most common type of bone cancer in children and adolescents.

The diagnosis and treatment of patients with osteosarcoma requires a multidisciplinary team approach. Resection of maxillary tumours remains a surgical challenge due to the possible aesthetic and functional secuelae.

We present herein the case of a 15 year-old female with an osteoblastic osteosarcoma of the left maxilla. It was treated with eight cycles of neoadjuvant chemotherapy, followed by a total left maxillectomy. Resection was performed through a modified Ferguson-Weber approach, using a titanium mesh to reconstruct the orbital base and the maxillary process. A palatal obturator was placed at the same time. The use of a three-dimensional model by stereolithography is extremely helpful in planning and performing the maxillectomy, as well as the facial reconstruction.
\end{abstract}

Key words: Osteosarcoma, maxillectomy, maxillofacial reconstruction. 


\section{Introduction}

Osteosarcoma is an osteoid-producing malignant mesenchimal tumor. In Mexico City, bone tumours account for the third most common cancer seen in adolescents and young adults, and the sixth most common malignant tumour in the younger population (1).

Worldwide, osteosarcoma is the most common primary malignant bone tumor, accounting for $5 \%$ of all tumours in children, and $9 \%$ of all sarcomas. Patients show a peak incidence related to puberty, between 10 and 14 years of age for girls, and between 15 and 18 years for boys (2). The rapid growth-rate during this time of life might explain its higher incidence in this age group. Less than ten percent of osteosarcomas occur in the head and neck, with mandibular and maxillary tumours being most common $(3,4)$.

Due to the high local recurrence rate, en-block complete resection with free surgical margins is essential for cure. Due to the anatomical limitations and subsequent cosmetic deformity, this is fairly difficult in tumours involving the facial bones.

Modern navigation systems allow the surgeon to identify the operative limits with precision, using a threedimensional model during the procedure. Computerassisted techniques can thus decrease the surgical risks and mortality.

The stereolithography creates a three-dimensional model with high accuracy. This enables the surgeon to exactly plan the areas of resection by determining the macroscopic limits of the lesion, and facilitates the conformation of the reconstructing titanium plate as well as the palatal obturator. It makes the technique safer, shortens the surgical time, and improves the patients' outcome by allowing a simultaneous maxillary reconstruction with better cosmetic and functional results.

The aim of the present work is show this technique of reconstruction in paediatric patients.

\section{Case reports}

We report herein the case of a 15 year-old female referred to us with an osteoblastic osteosarcoma in the left upper maxilla. No metastatic disease was found on extension studies.

On physical examination, the patient presented a $4 \mathrm{~cm}$ mass in the left maxillary sinus. The mass was firm and well circumscribed, and produced a mild displacement of the ocular globe (Figure 1a). It extended intraorally into the left palate as an ulcerated, painful mass, not crossing the midline, (Figure 1b).

After eight cycles of neoadjuvant chemotherapy, a CTscan revealed stable tumoural activity (Figures $1 \mathrm{c}$ and 1d). Stereolithography (Figures 1e and 1f) was then performed to adequately plan for the surgical resection and reconstruction.
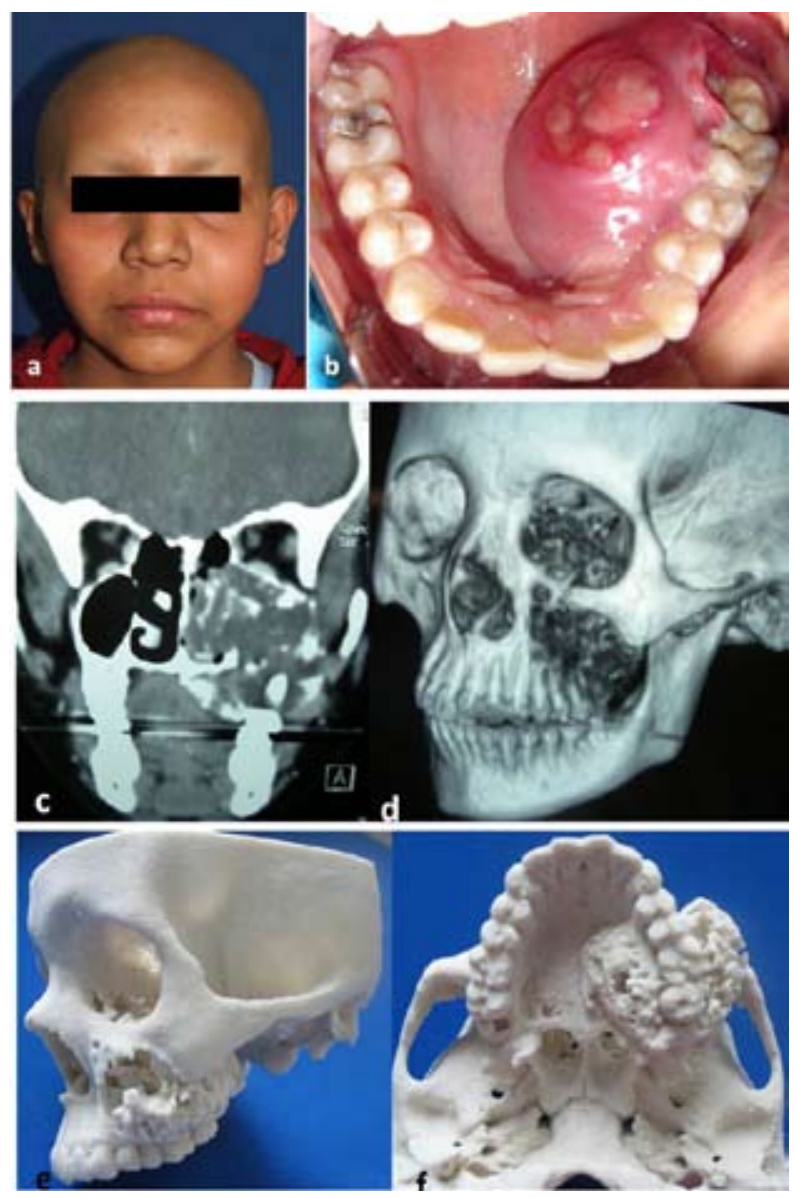

Fig. 1. Clinical images: a) Clinical images: Left maxillary mass in a 15-year-old female with mild displacement of the eye. b) Clinical images: Ulcerated mass extending into the left hard palate. c) Imaging technique: Coronal head CT demonstrates the mass originating in the left upper maxilla, invading the base of the orbit, the nasal cavity and the hard palate, d) Imaging technique: CT 3-D reconstruction showing the mass in the left upper maxilla, invading the base of the left orbit and the nasal cavity. e) Sterolithography technique: Lateral view of a stereolithography reconstruction of the patients' skull demonstrates the extent of the mass, f) Sterolithography technique: Inferior aspect of stereolithography reconstruction depicting the maxillary osteosarcoma involving the left alveolar process and the hard palate.

\section{Surgical Technique:}

Under general anaesthesia with naso-tracheal intubation, we performed a modified Ferguson-Weber approach (Figure 2a), completing the incision at the left gingivolabial border up to the second molar, in order to create a complete facial flap including skin, subcutaneous tissues, and facial expression muscles and nerves (Figure $2 b)$. The tumour was resected "en-block" including the nasal process, base of the orbit, zygomatic arch, palate, and pterigoid fossa (Figures $3 \mathrm{a}$ and $\mathrm{b}$ ). The defect was then reconstructed with a titanium mesh (previously conformed on the plaster model) for the base of the orbit and the malar bone (Figure 3c), and a palatal obturator 


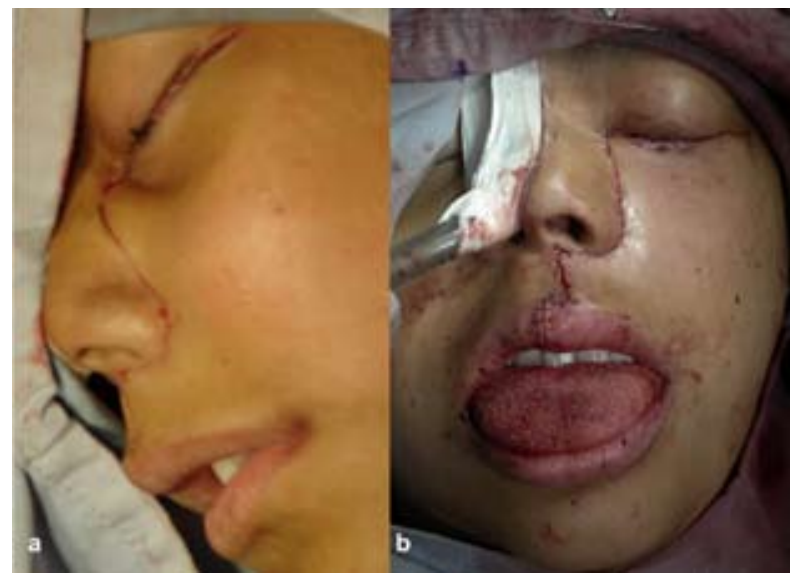

Fig. 2. Surgical approach: a) Modified Ferguson-Weber approach with naso-tracheal intubation. The incision begins at the inferior eyelid line, continuing lateral to the nose and around the alae, coming down through the lip pilar and into cupid's bow, cutting the lip at the midline, and continuing on the gingivo-labial mucosal fold up to the level of the second upper molar, b) Immediate postoperative appearance with the facial flap closed in layers, and the preoperatively designed palatal obturator in place. constructed preoperatively (Figure $3 \mathrm{~d}$ ).

The patient had an uneventful recovery and was discharged from the hospital on postoperative day 7 , in good general conditions, tolerating diet and with adequate facial expression.

The patient then received radiotherapy for local control and several cycles of adjuvant chemotherapy. She unfortunately suffered a relapse nine months after the procedure, for which she underwent a re-excision of the tumour and continued adjuvant chemotherapy.

She is alive and well 18 months later, with no physiological secuelae, and following the standard follow-up protocol.

\section{Discussion}

According to their histology, osteosarcomas can be osteoblastic (50\% of cases), chondroblastic (resembling cartilage $-25 \%$ ), and fibroblastic (the remaining $25 \%$ of cases) (4).

Osteosarcomas are highly malignant tumours. Eighty percent of patients are considered to have micro-metastatic disease at diagnosis, and ninety percent of clinical

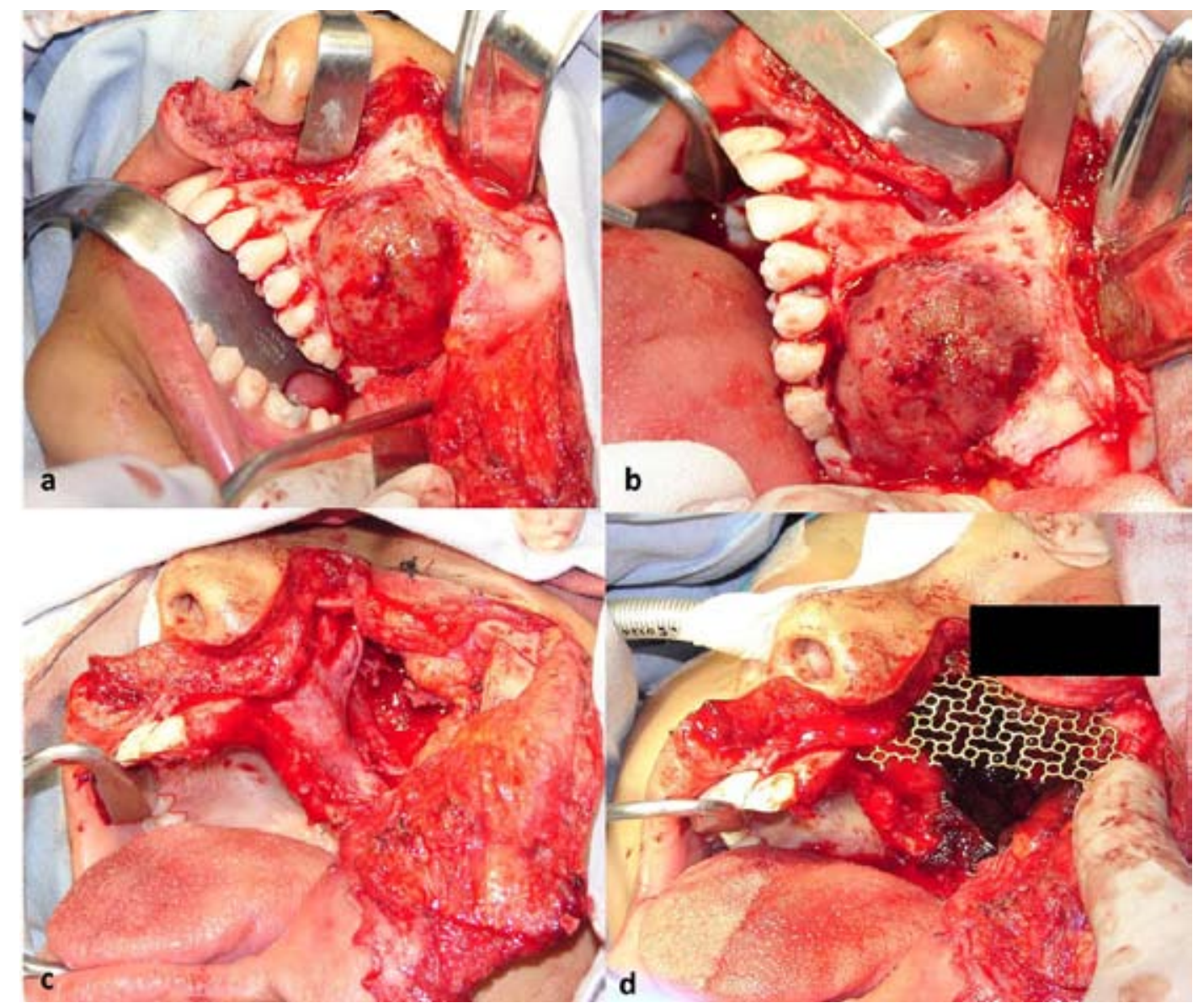

Fig. 3. Surgical technique: a) Facial flap including skin, subcutaneous tissue, muscles and facial nerve in order to preserve facial expression. The tumor is seen destroying the periostium of the left upper maxilla, $b$ ) The tumor is being resected "enblock" by cutting the maxilla with a saw, with free margins around the mass. Measurements for the exact cut where taken from the stereolithography model, c) Complete left maxillectomy demonstrates the nasopharynx and the cut through the midline in the palate, d)An L-shaped titanium mesh has been placed to reconstruct the base of the orbit as well as the malar process. 
metastases occur in the lungs (5).

A plain film can suggest the diagnosis with lythic, blastic or mixed bone lesions, cortical destruction, and soft tissue involvement.

CT-scan and MRI are useful to define the tumour extension within the bone, as well as into the surrounding soft tissues and neurovascular bundles (6).

Since the 1970's chemotherapy has improved survival by managing undetected micro-metastatic disease, creating tumour necrosis, and allowing complete resections after tumour shrinkage and decreased tumour vascularity (6).

Using data from the CT-scan or MRI, stereolithography creates a 1:1 size three-dimensional model of the patient's skull $(7,8)$. Models are reproduced with $99 \%$ accuracy (9), thus, they have proved to be invaluable to limit the margins of resection, conforming the maxilloorbital plate, and designing the palatal obturator.

Resection of maxillary tumours in paediatric patients might produce important anatomic, physiologic (swallowing, mastication and phonation), and psychological secuelae (10). It also produces fluid exchange between the mouth and the nasal cavity, with a consequent bacterial normal flora change. Food residue in the nasal cavity presents a risk for aspiration.

These problems can be palliated by using palatal obturators which improve the patients quality of life and psychological well-being (10). Palatal obturators ought to be low-weight, stable, comfortable, hypoallergenic, and capable of remodelling the face contour as well as the lost physiologic functions for the patient.

Our case shows step by step an example of this technique.

In conclusion Osteosarcomas should be treated at referral centres, by a multidisciplinary team (Oncologists, maxillofacial and head and neck surgeons, radiotherapist, maxillofacial prosthodontist and plastic surgeons) with experience managing these lesions. Modern technology enables us to create actual size, three-dimensional models of the patients' skull. The procedure can then be better planed, favouring complete tumour resection, and simplifying the maxillofacial reconstruction.

\section{References}

1. Estrada-Villaseñor E, Delgado Cedillo EA, Rico Martínez G. Prevalence of bone neoplasms in adolescents and young adults. Acta Ortop Mex. 2008; 22:316-20.

2. Heare T, Hensley MA, Dell'Orfano S. Bone tumors: osteosarcoma and Ewing's sarcoma. Curr Opin Pediatr. 2009; 21:365-72.

3. Tran LM, Mark R, Meier R, Calcaterra TC, Parker R. Sarcomas of the Head and Neck.Pronostic factors and treatment strategies. Cancer 1992;70:169-77.

4. Huvos AG. Osteosarcoma in adolescents and young adults: new developments and controversies. Commentary on pathology. Cancer Treat Res. 1993;62:375-7.

5. Neifeld JP, Michaelis LL, Doppman JL. Suspected pulmonary metastases. Correlation of chest X-ray, whole lung tomograms and operative findings. Cancer 1977;39:383-7.
6. Schreinman JS, Crass JR, Wick MR, Maile CW, Thompson RC. Osteosarcoma role of CT in limb-sparing treatment. Radiology. 1986. 161:485-8

7. Whelan J, Seddon B, Perisoglou M. Management of Osteosarcoma. Curr Treat Options Oncol. 2006; 7:444-53

8. Ono I, Gunji H, Suda K, Kaneko F. Method of preparing an exact size model using helical volume scan computed tomography. Plast Reconst Surg 1994; 93: 1363-71.

9. Sinn DP, Cillo JE Jr, Miles BA. Stereolithography for craniofacial surgery. J Craniofac Surg. 2006; 17:869-75.

10. Rogers S, Lowe D, McNally D,Brown JS, Vaugha ED. Healthrelated quality of life after maxillectomy: a comparison between prosthetic obturation and free flap. J Oral Maxillofac Surg 2003; 61: 174-81. 